\title{
Jets in deep-inelastic scattering at HERA
}

\author{
Matthew Wing用 \\ (on behalf of the $\mathrm{H1}$ and ZEUS collaborations) \\ University of Bristol, DESY, Notkestrasse 85, 22607 Hamburg, Germany
}

\begin{abstract}
Jet cross sections in deep-inelastic scattering over a wide region of phase space have been measured at HERA. These cross section measurements provide a thorough test of the implementation of Quantum Chromodynamics in next-toleading order (NLO) calculations. They also provide the opportunity to test the consistency of the gluon distribution in the proton as extracted from (mainly) inclusive DIS measurements. Comparison of the cross sections with NLO enables accurate extractions of the strong coupling constant, $\alpha_{s}$, to be made, several of which are reported here.
\end{abstract}

\section{Introduction}

Deep-inelastic ep scattering (DIS) at HERA, in which a photon of virtuality, $Q^{2}$, is exchanged, can lead to the production of jets in the final state. Crosssection measurements of these types of processes provide stringent tests of the perturbative Quantum Chromodynamics (pQCD) formalism in next-to-leading order (NLO) calculations, the structure of the proton and allow measurements of the strong coupling constant, $\alpha_{s}$. These features can be seen in the formula for the cross section, $d \sigma$, which is factorised into a convolution of the hard partonic cross section, $d \hat{\sigma}_{a}\left(x, \alpha_{s}\left(\mu_{R}^{2}\right), \mu_{R}^{2}, \mu_{F}^{2}\right)$, and the proton's parton density, $f_{a}\left(x, \mu_{F}^{2}\right)$ :

$$
d \sigma=\sum_{a=q, \bar{q}, g} \int d x f_{a}\left(x, \mu_{F}^{2}\right) d \hat{\sigma}_{a}\left(x, \alpha_{s}\left(\mu_{R}\right), \mu_{R}^{2}, \mu_{F}^{2}\right) .
$$

The hard partonic cross section is a power series expansion in $\alpha_{s}$ and is calculable in pQCD. The proton's parton density is derived from fits to published data, such as inclusive DIS measurements [1] and high transverse energy jet production in $p \bar{p}$ production [2].

In the region of momentum fraction, $x$, and $Q^{2}$ in which DIS jet measurements are currently performed, the quark density in the photon is well constrained from particularly inclusive DIS measurements. At high $Q^{2}$ where the quark initiated process, QCD Compton (see figure 1(a)), is dominant, the jet cross section measurements test pQCD. At lower $Q^{2}$, where the boson-gluon fusion process (see figure 1 1 (b)) is dominant, measurements of the jet cross sections provide complementary information on the gluon

† wing@mail.desy.de 
density in the proton, which from DIS is only constrained through the interpretation of scaling violations in NLO fits.

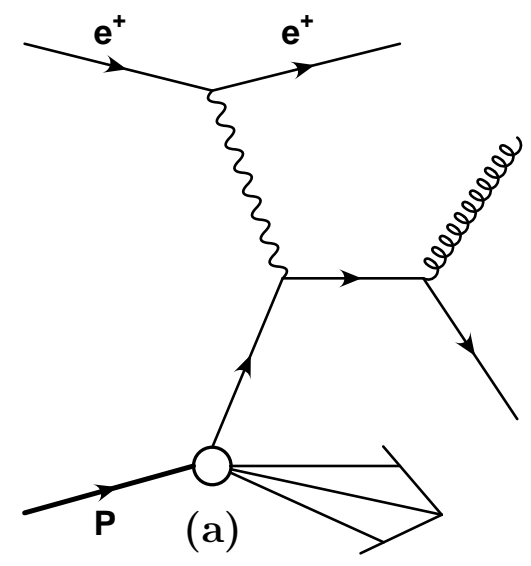

(a)

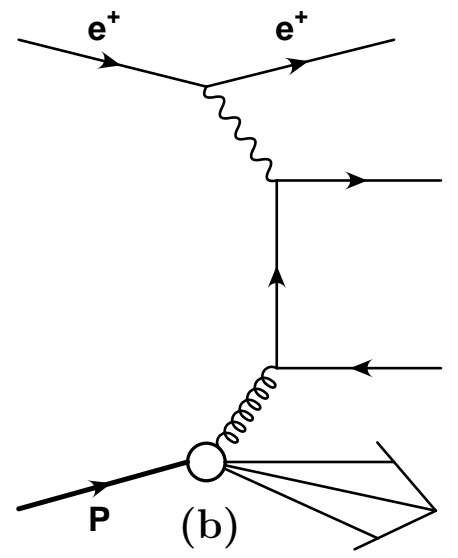

(b)

Figure 1. (a) The QCD Compton and (b) boson-gluon fusion processes.

In this paper, the latest measurements from both the $\mathrm{H} 1$ and ZEUS collaborations on DIS jet production will be discussed. Initially the jet cross section comparisons with NLO will be shown and then the extraction of the gluon density and $\alpha_{s}$ will be presented.

Limitations of both the current data and theory and some of their possible solutions will be addressed. In particular, the measurements raise the following issues:

- at which points are the theoretical or experimental errors dominant?

- which of the two "natural" scales, $Q$ and transverse energy, $E_{T}$, is the more appropriate?

- is there a region where the DGLAP [3] formalism breaks down and BFKL [4] is more appropriate?

- is there an effect of the resolved photon?

\section{Inclusive jet measurements}

The two inclusive jet measurements presented here have different goals; the first is concentrated in a "safe" region to test pQCD and extract $\alpha_{s}$, whilst the second extends the kinematic phase space to more extreme regions to look for suggestions of BFKL effects. The safe region was defined by considering jets at a reasonably high $-Q^{2}$ of greater than $125 \mathrm{GeV}^{2}$, high $-E_{T \text {,jet }}^{\mathrm{B}}$ of greater than $8 \mathrm{GeV}$ in a central region of the detector, $-2<\eta_{\text {jet }}^{\mathrm{B}}<1.8$, where the jets are reconstructed in the Breit frame of reference. To be more sensitive to BFKL effects, a second region was probed; $5<Q^{2}<100 \mathrm{GeV}^{2}, E_{T, \text { jet }}^{\mathrm{B}}>8 \mathrm{GeV}$ and $-1<\eta_{\text {jet }}^{\mathrm{LAB}}<2.8$.

The measured cross section as a function of $Q^{2}$, for $Q^{2}>125 \mathrm{GeV}^{2}$, is shown in figure 2(a) compared to predictions from NLO QCD implemented in the DISENT [5] 
program. The data fall by five orders of magnitude and are reasonably well described by the predictions corrected for hadronisation effects and using two different values for the renormalisation scale, $\mu_{R}$. The description is quantified in figure 2(b) where the ratio of the data to the prediction (using $\mu_{R}=Q$ ) is shown. The data lie above the theory by about $12 \%$ at $Q^{2}<500 \mathrm{GeV}^{2}$, although the size of the theoretical and experimental uncertainties rule out any firm conclusions. At higher $Q^{2}$, the prediction describes the data well. Similar results were observed when considering the cross section as a function of the transverse energy of the jet. The data can therefore be used to extract a value of $\alpha_{s}$, which was done at high $-Q^{2}$ and high $-E_{T, \mathrm{jet}}^{\mathrm{B}}$. As can be seen in figure 2(b), extracting $\alpha_{s}$ at $Q^{2}>500 \mathrm{GeV}^{2}$ leads to a smaller theoretical uncertainty, arising mainly from the variation of the renormalisation scale by a factor of two.
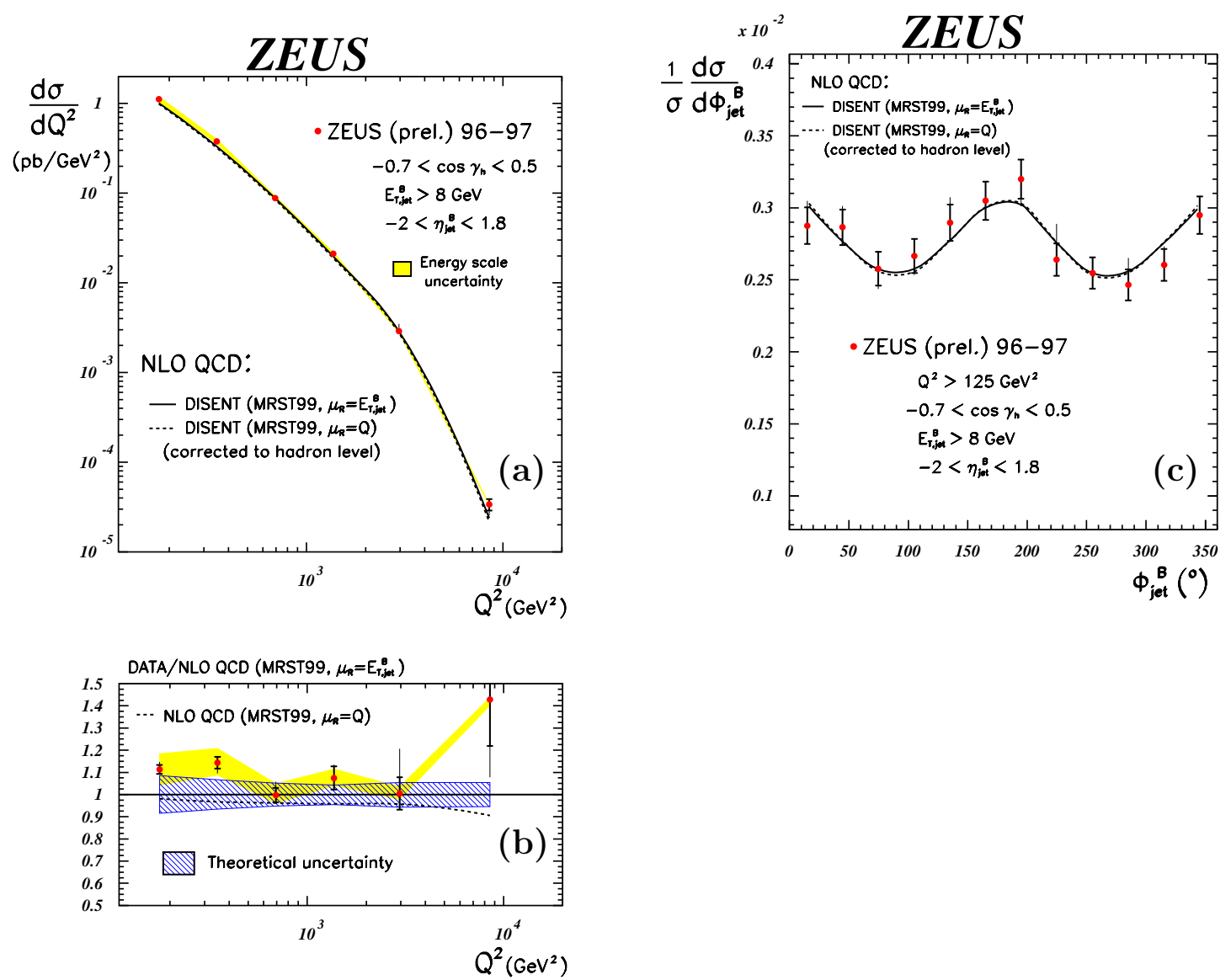

Figure 2. Inclusive jet cross sections as a function of (a) $Q^{2}$ and (b) the ratio of data to NLO and (c) the normalised cross section as a function of $\phi_{\text {jet }}^{\mathrm{B}}$. The data are shown as the points with statistical errors (inner bars) and statistical and systematic errors added in quadrature (outer bars). The NLO is shown with two different renormalisation scales; $E_{T, \text { jet }}^{\mathrm{B}}$ (solid line) and $Q$ (dashed line).

A further test of QCD is the structure of the cross section in the azimuthal angle, $\phi_{\text {jet }}^{\mathrm{B}}$, which is defined as the angle between the lepton scattering plane and the jet 
production plane. The cross section is predicted to have the form [6],

$$
\frac{d \sigma}{d \phi_{\text {jet }}^{\mathrm{B}}}=A+B \cos \phi_{\text {jet }}^{\mathrm{B}}+C \cos 2 \phi_{\text {jet }}^{\mathrm{B}},
$$

and has not be seen before in neutral-current DIS jet production. The effect has been seen in charged hadron production in DIS [0]. Without tagging the nature of the final state jets, the cross section is expected to reduce to:

$$
\frac{d \sigma}{d \phi_{\text {jet }}^{\mathrm{B}}}=A+C \cos 2 \phi_{\text {jet }}^{\mathrm{B}}
$$

The measurement is shown in figure 2(c) compared to the NLO predictions. The form of Eq. (3) is clearly seen in the data for the first time in neutral current DIS jet production and the calculation gives a good description of the shape.

Comparisons of inclusive jet data at lower scales and for more forward-going jets with NLO are shown in figure 3. The data are shown in different regions of pseudorapidity of the jet so as to maximise sensitivity to BFKL effects which are expected to be largest at forward values. The NLO calculation has been evaluated using two different choices of scale; $E_{T}$ and $Q$, shown in figures $3($ a) and $3(\mathrm{~b})$, respectively.
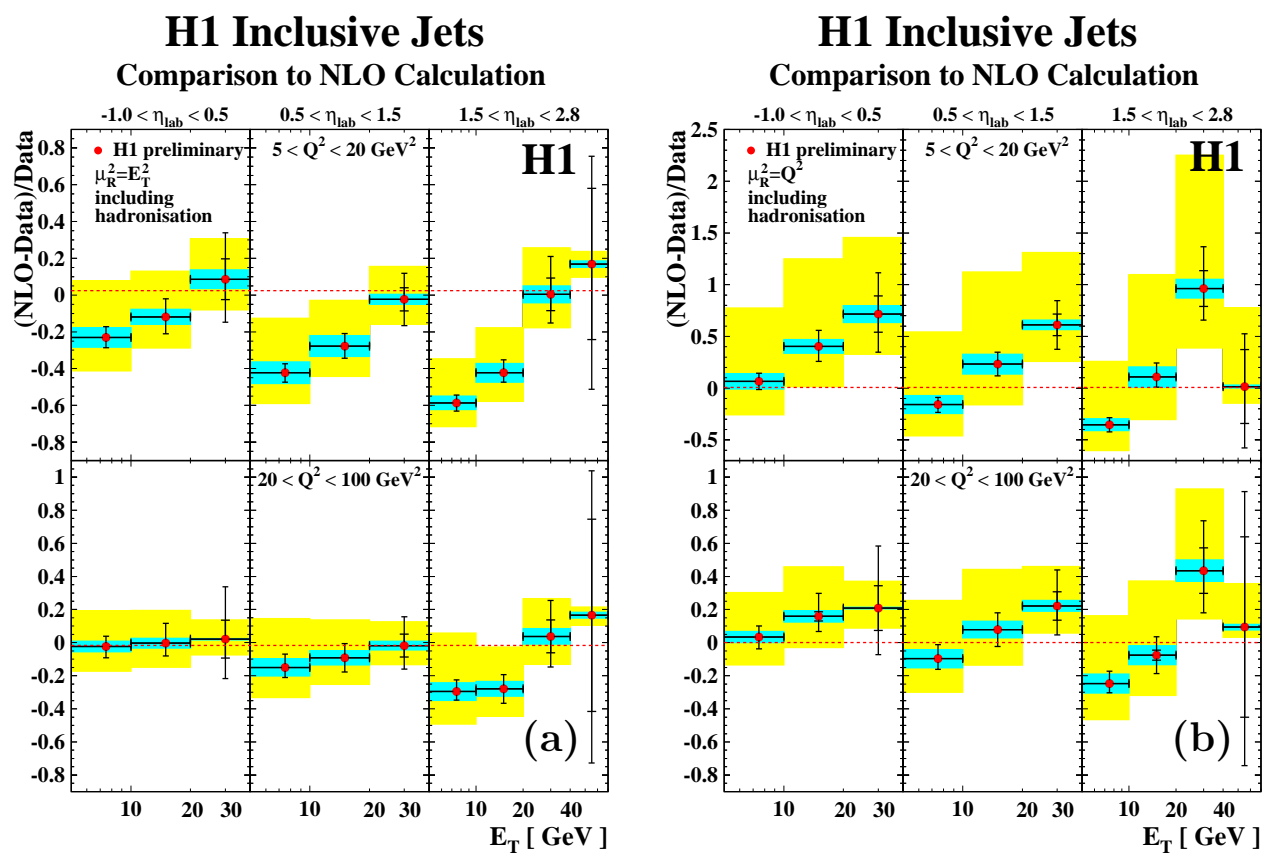

Figure 3. Difference between data and NLO as a function of the transverse jet energy, when using the renormalisation scale set to (a) $E_{T}$ and (b) $Q$. The data are shown as the points with statistical errors (inner bars) and statistical and systematic uncertainties added in quadrature (outer bars). The NLO is displayed with the hadronisation (inner band) and renormalisation scale (outer band) uncertainties.

Overall, the predictions generally describe the data, although with very large uncertainties of sometimes up to $50-100 \%$. The agreement is better at higher $Q^{2}$ 
and at lower $Q^{2}$ it is better when using $Q$ rather than $E_{T}$ as the scale in the calculation. It should be noted that at these low values of $Q^{2}, E_{T}>Q$. When using $E_{T}$ as the scale, the NLO does poorly in the forward region at low values of transverse energy. This is exactly the region where BFKL effects are expected to show up. However, the large uncertainties on the DGLAP-based calculation shown, preclude any statement of the need for BFKL effects in the measurement. These large uncertainties in the theory, and hence a significant difference between NLO and the next higher order, are probably not surprising given the LO and NLO cross sections differ by up to a factor of ten at low $E_{T}$ and forward $\eta_{\mathrm{jet}}^{\mathrm{LAB}}$. Clearly this kind of measurement needs more accurate calculations in order to search for a breakdown of the DGLAP formalism.

\section{Dijet measurements}

Along with BFKL effects, at low $-Q^{2}$ the existence and nature of a resolved photon may also play a rôle. The resolved photon is expected to be important when the transverse energy of the outgoing jets, $E_{T}$ is much greater than $Q$, whereas BFKL effects are expected to be most significant when the two quantities are roughly equal. The rôle of the resolved photon has been studied in dijet production with $5<Q^{2}<15 \mathrm{GeV}^{2}$ and at least two jets, such that again $E_{T}>>Q$. The ratio of the dijet data as a function of the momentum fraction, $x_{\mathrm{B}}$, is poorly described by the calculation when the renormalisation scale is set to the intuitive hard scale, $\sim E_{T}$. When choosing the scale to be $Q$, the prediction is higher and describes the data well, but suffers from a lack of predictive power due to its large uncertainty. Therefore, using the natural scale, the impact of the resolved photon was studied as implemented in the calculation JETVIP [8]. The inclusion of a resolved photon into the calculation compared to the data is shown in figure 4 . It can be seen that the calculation lies below the data at low $-x_{\mathrm{B}}$ and that

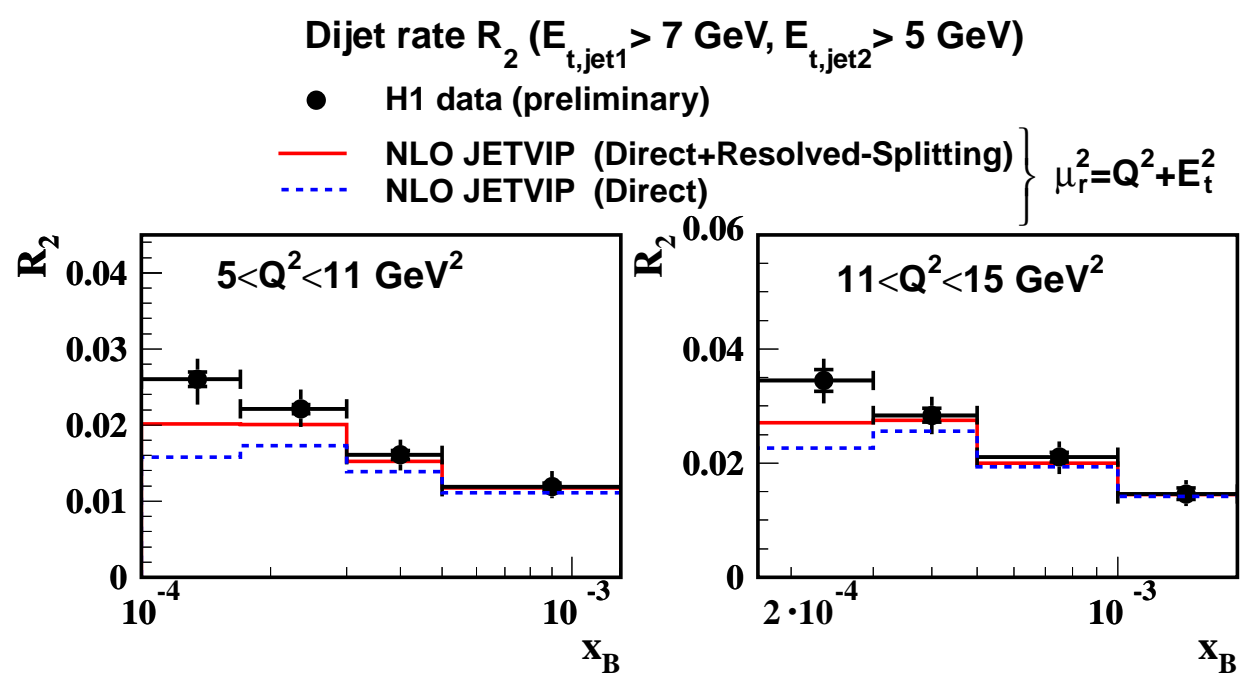

Figure 4. Ratio of cross sections for dijet and inclusive production as a function of $x_{\mathrm{B}}$. The data are shown as in figure 3. The NLO calculation is shown with (solid line) and without (dashed line) a resolved component. 
the description improves with the inclusion of a resolved photon. The effect is larger at the lower range in $Q^{2}$, but is not enough to completely describe the data. Due to large uncertainties in the calculations and unknowns in the underlying physics processes, fundamental parameters such as $\alpha_{s}$, are measured at higher values of $Q^{2}$ or $E_{T}$, where the uncertainties are reduced.

The dijet cross section at slightly higher $E_{T}$ is shown as a function of $Q^{2}$ in figure 5 . Note that here the jets are reconstructed in the Breit frame whereas in figure 1 they

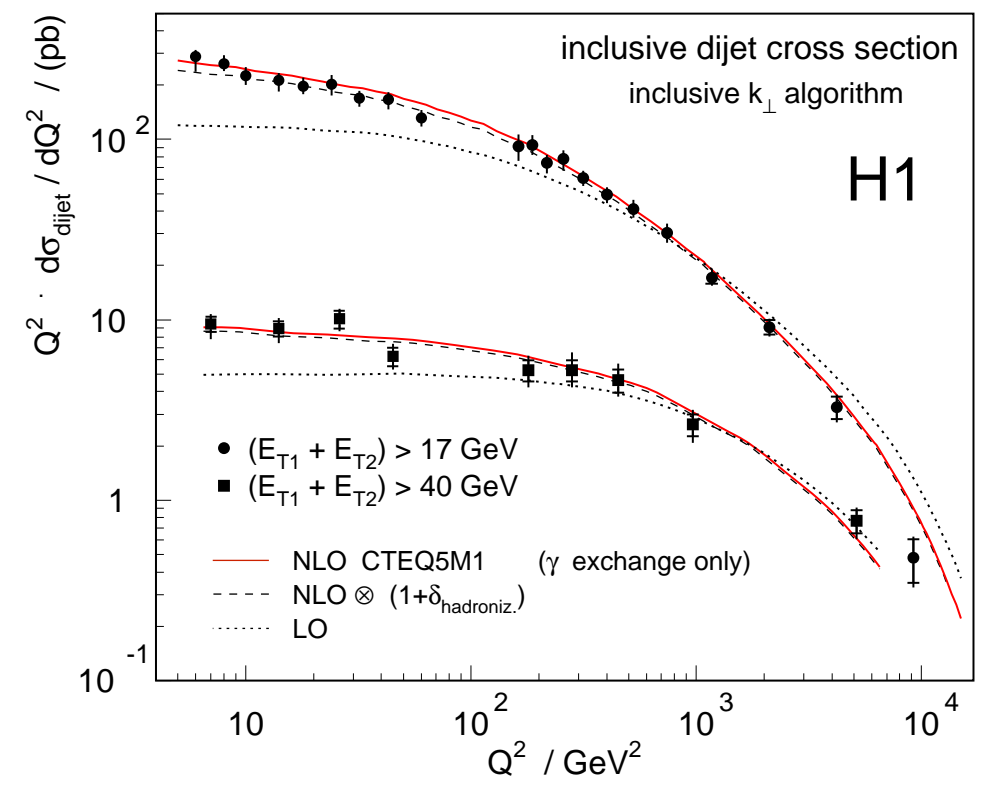

Figure 5. Inclusive dijet cross section as a function of $Q^{2}$ for two regions of the sum of the transverse energies of the highest $E_{T}$ jets.

were in the hadronic centre-of-mass frame. The description of the data by the NLO calculation in figure 5 is good over the whole range in $Q^{2}$. Both data and theory also scale similarly with different minimum transverse energy requirements. The size of the LO to NLO corrections are reasonably large at low $-Q^{2}$ and the hadronisation corrections are also significant. These factors again demonstrate the need to consider higher values of $Q^{2}$ for the extraction of fundamental parameters. The description of other variables (not shown) by the NLO is also generally good.

The dijet cross section at high- $Q^{2}$, greater than $470 \mathrm{GeV}^{2}$, is shown in figure 6 along with the inclusive cross section and dijet rate. All measurements are well described by the NLO calculation. The dijet rate is a particularly good variable to measure as it is sensitive to $\alpha_{s}$ (as demonstrated in figure 6(b)) and experimental and theoretical uncertainties are expected to somewhat cancel. Cancellation of some of the theoretical uncertainty can be seen in figure 0 where the size of the uncertainty related to the parton density function is shown. This was estimated using the program EpDFLIB [9]. This program propagates the statistical and systematic uncertainties of each data set used in the PDF, MBFIT and provides additional PDF sets to quantify the theoretical 
uncertainties. Figure 0 shows that the total uncertainty arising from the PDF for the rate is $1.5 \%$, smaller than for both the inclusive $(2.5 \%)$ and dijet (4\%) cross sections.

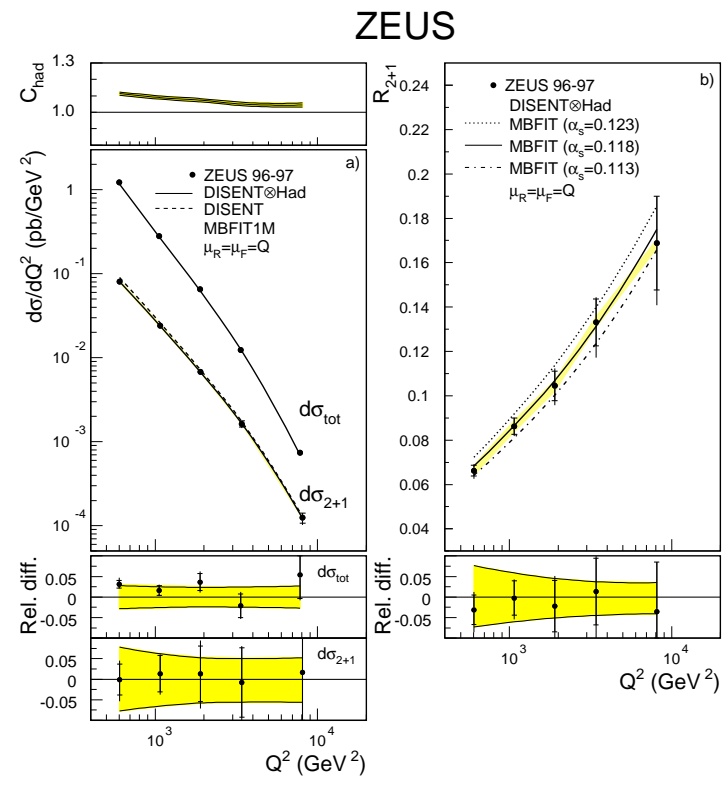

Figure 6. (a) Inclusive and dijet cross sections in DIS as a function of $Q^{2}$ and (b) the ratio of the two. The data are compared to NLO and the relative difference between them is also shown.

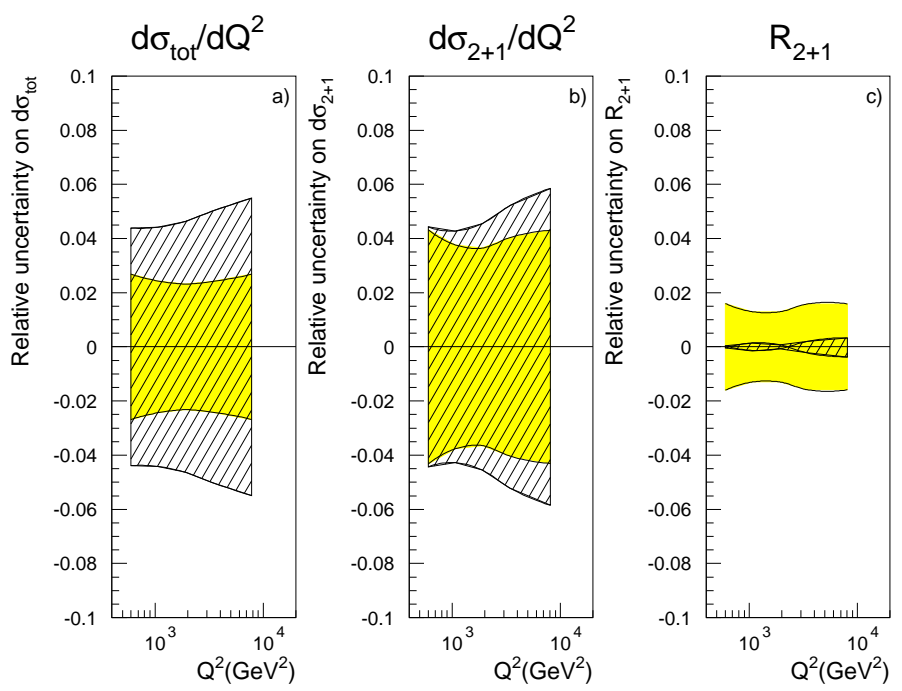

Figure 7. The relative uncertainty on a) the inclusive, b) the dijet differential cross sections and c) the dijet fraction, due to the statistical and systematic uncertainties of each data set used in the determination of the MBFIT PDFs. The shaded and hatched bands indicate the uncertainties obtained taking into account and not taking into account the correlations among the PDF's parameters. 


\section{Measurements of three jet production}

Neutral current DIS with three jets in the final state has recently been measured which, with a more complex final state, has the potential to provide a more stringent test of pQCD. A new NLO three-jet calculation [10] allows the comparison to be made for the first time. The ratio of three- to two-jet cross sections can also be measured. As with the ratio of cross sections of two-jet and inclusive production, it is expected that there will be cancellation of some experimental and theoretical uncertainties. Again, this measurement should allow an accurate extraction of $\alpha_{s}$, with much reduced uncertainties.

In figures 8 and $\mathrm{g}(\mathrm{a})$, the cross-section measurements are shown as a function of Bjorken-x, $x_{\mathrm{Bj}}$, three-jet mass, $M_{3 \mathrm{jet}}$ and $Q^{2}$. Predictions to LO and NLO, both corrected for hadronisation effects, are compared to the measurements. The addition of the NLO corrections are significant, particularly at low- $x_{\mathrm{Bj}}$ and $Q^{2}$ and low mass, and their inclusion into the prediction provides a good description of the data. Also shown, in figure $\mathrm{g}(\mathrm{b})$, is the ratio of data to theory as a function of $Q^{2}$. The NLO can be seen to describe the data to within about $10 \%$ over the whole region in $Q^{2}$. The uncertainty due to varying the value of the renormalisation scale is up to $30 \%$ at low- $Q^{2}$ and decreases with increasing $Q^{2}$. The uncertainty arising from the value of $\alpha_{s}$ and the gluon content of the proton are reasonably constant with $Q^{2}$ and are about $20 \%$ and $10 \%$, respectively.
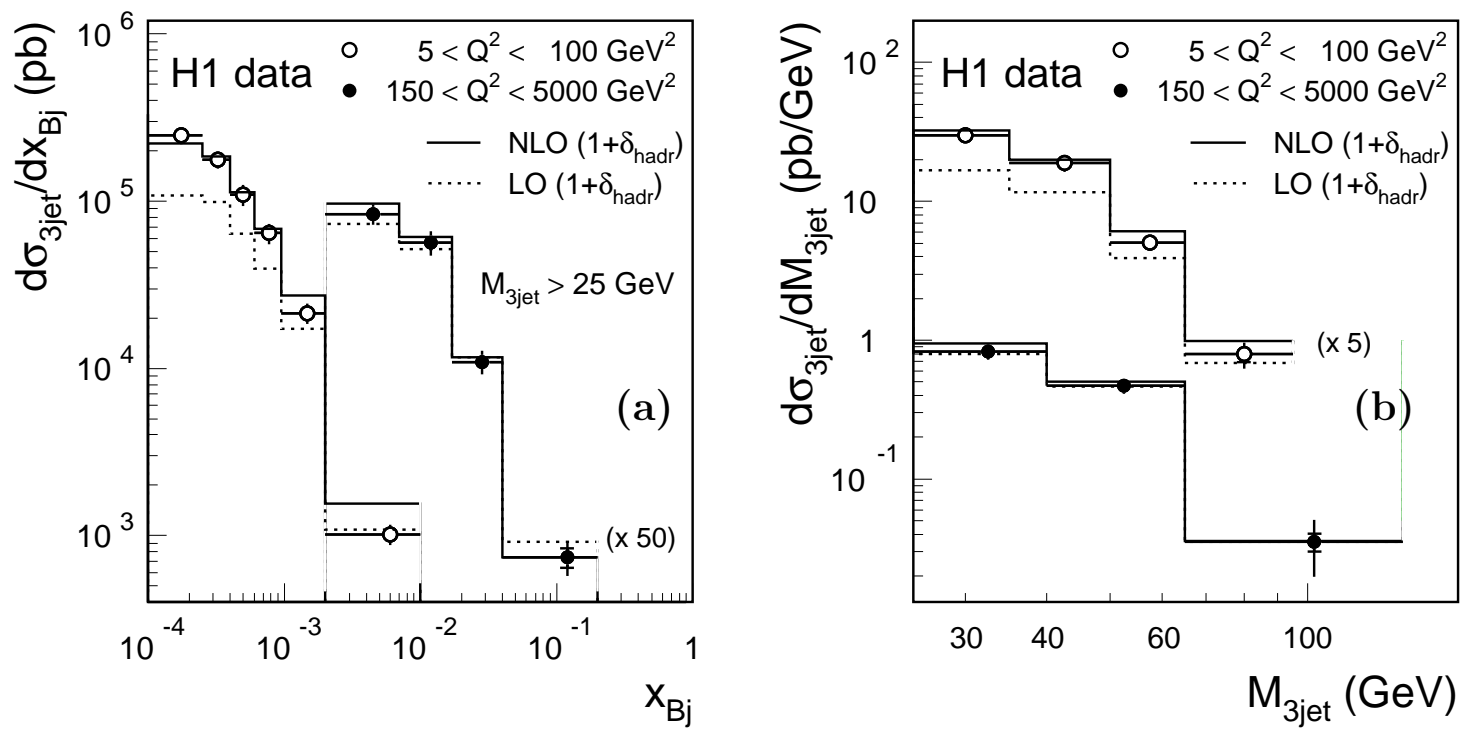

Figure 8. The inclusive three-jet cross section measured as a function of (a) $x_{\mathrm{Bj}}$ and (b) $M_{3 j \mathrm{jet}}$. The predictions of QCD are shown at LO (dotted line) and NLO (solid line), both corrected for hadronisation effects.

The ratio of three- to two-jet cross sections is also shown in figure 9(c) as a function of $Q^{2}$. The NLO again describes the data well and, as expected, the theoretical uncertainties are significantly reduced. In particular, the uncertainty due to the 
renormalisation scale and gluon PDF are both considerably smaller than the uncertainty arising from varying $\alpha_{s}$. This demonstrates the potential of the distribution in providing an accurate measurement of $\alpha_{s}$. This measurement awaits an increased event sample to realise its full potential.
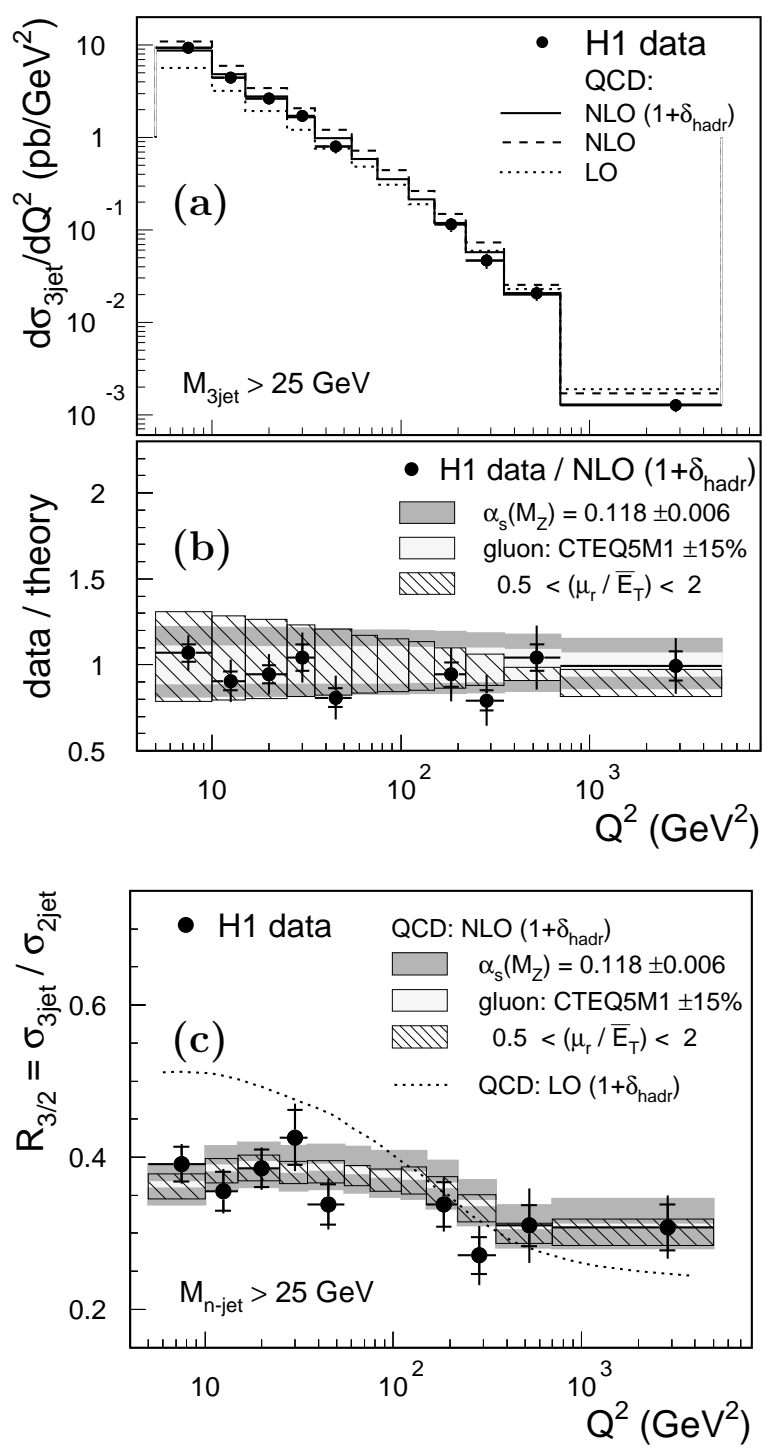

Figure 9. (a) The inclusive three-jet cross section measured as a function of $Q^{2}$. The predictions of QCD are shown at LO (dotted line) and NLO (solid line), both corrected for hadronisation effects. (b) The ratio of the cross sections in data and theory and the associated theoretical uncertainties. (c) The ratio of the three- to two-jet cross section and the associated theoretical uncertainties.

\section{Measurements of jet substructure}

Measurements of jet substructure in NC DIS have been performed and used to test pQCD and perform extractions of $\alpha_{s}$. Measurements of both the integrated jet shape 
and mean subjet multiplicity in inclusive jet NC DIS were made. The lowest nontrivial-order contribution to these quantities is given by $\mathcal{O}\left(\alpha \alpha_{s}\right)$ pQCD calculations. Thus measurements of the jet shape and subjet multiplicity provide a stringent test of pQCD calculations beyond LO and allow a determination of $\alpha_{s}$. Subjets were resolved within a jet by considering all particles that are associated with it and by repeating the application of the $k_{T}$ cluster algorithm 11 until, for every pair of particles $i$ and $j$, the quantity $d_{i j}$ was above $d_{\text {cut }}=y_{\text {cut }} \cdot\left(E_{T}^{\text {jet }}\right)^{2}$. All remaining clusters were called subjets. The subjet structure depends upon the value chosen for the resolution parameter, $y_{\text {cut }}$.

In figure 10, the measurements of mean subjet multiplicity are shown as a function of the resolution scale, $y_{\text {cut }}$, and $E_{T}^{\text {jet }}$ at a fixed value of $y_{\text {cut }}$ equal to 0.01. Also shown are the predictions from LO and NLO QCD, corrected for effects of hadronisation. The NLO predictions give a good description of the data for all values of $y_{\text {cut }}$ and $E_{T}^{\text {jet }}$ and can therefore be used to perform a measurement of $\alpha_{s}$. Similar results are also seen for the measurements of the jet shape.
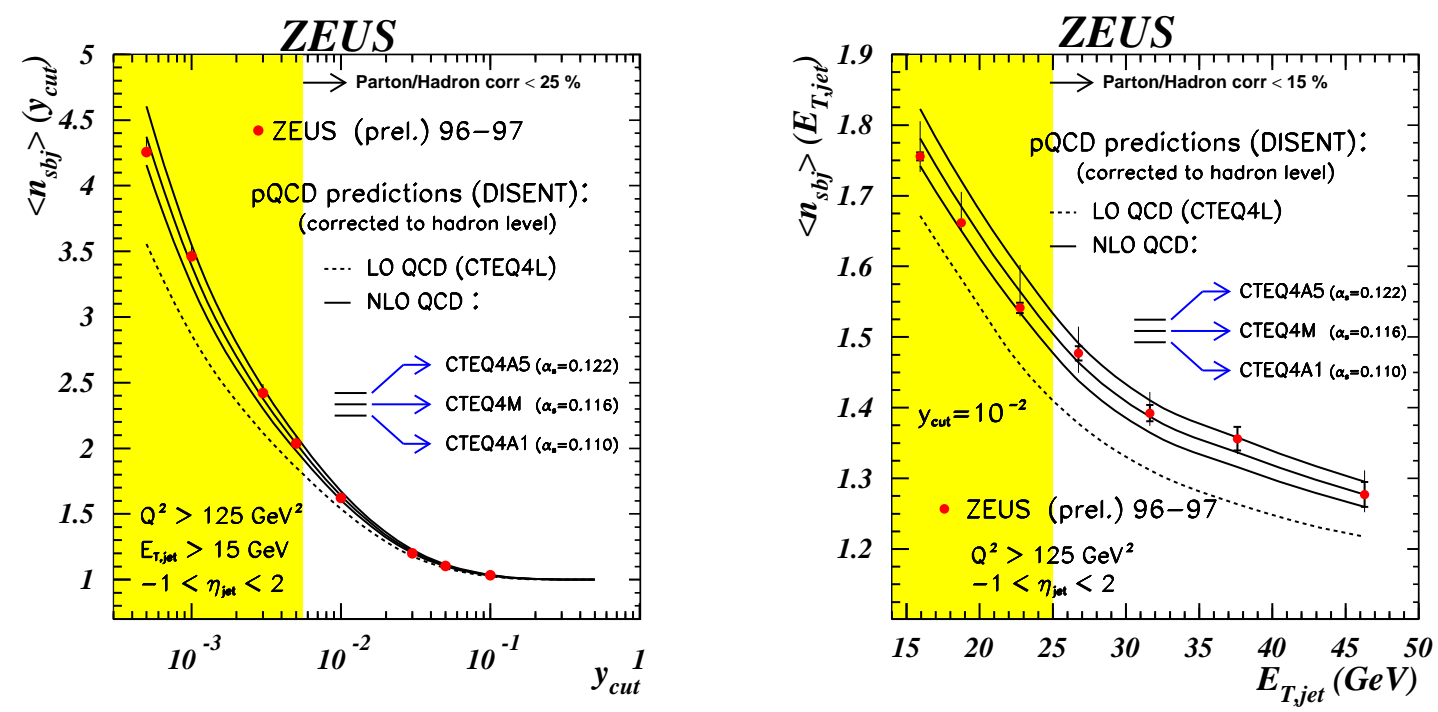

Figure 10. The mean subjet multiplicity, $\left\langle n_{\mathrm{sbj}}>\right.$ as a function of $y_{\mathrm{cut}}$ and $E_{T}^{\text {jet }}$ for fixed $y_{\text {cut }}$. The data points are compared to pQCD predictions to LO and NLO, both corrected for hadronisation effects.

\section{The gluon density in the proton}

As stated in section 1, jet production at HERA is directly sensitive to the gluon content of the proton at LO. Therefore, the measurements shown here can in principle be used to constrain the gluon density, complementing the extractions from (predominantly) measurements of the proton structure function, $F_{2}^{p}$. This has been performed by simultaneously fitting the inclusive and dijet cross sections and the measurements from inclusive DIS. The result of this fit are shown in figure 11, where the extraction 
is compared to other parametrisations of the gluon PDF. The extraction from this simultaneous fit is consistent with all other parametrisations shown. The effect of the jet data in further constraining the gluon density would be enhanced by using the data at lower $Q^{2}$. However, due to the large theoretical uncertainties, arising predominantly from varying the value of the renormalisation scale, an accurate extraction of the gluon density from jet data remains limited. Also shown in figure 11 is the result of simultaneously constraining both the gluon density and measuring $\alpha_{s}$. The data are sensitive to the product $\alpha_{s} \cdot x g(x)$, but do not permit a precise determination of both simultaneously.
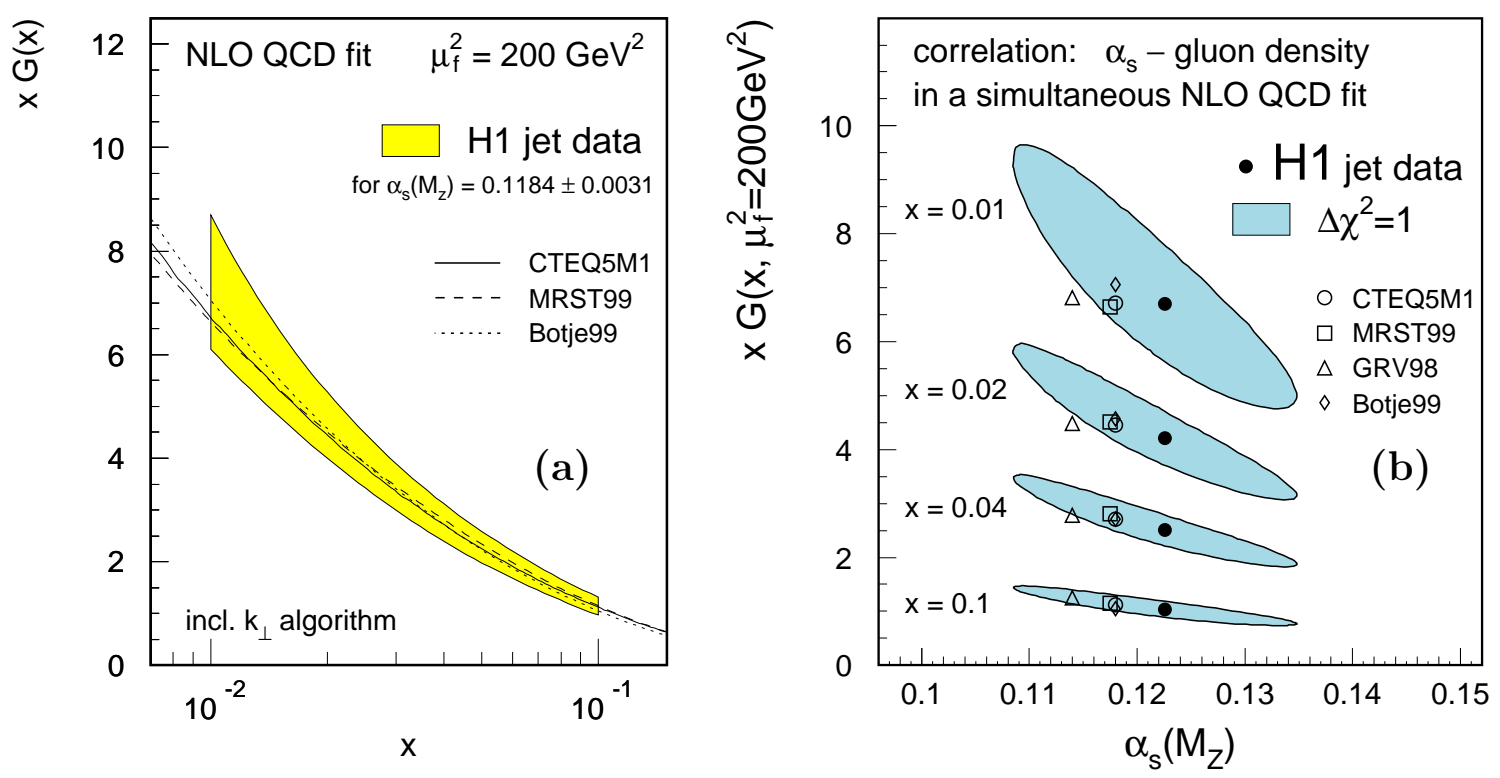

Figure 11. (a) The gluon density, $x G(x)$, in the proton, determined in a simultaneous fit to the inclusive-jet and dijet cross sections and the inclusive DIS cross section. The error band includes experimental and theoretical uncertainties. (b) Correlation of simultaneously constraining the gluon density and measuring $\alpha_{s}$.

\section{Measurements of $\alpha_{s}$}

As shown in the previous sections, measurements of jet cross sections and jet substructure provide an opportunity to extract a value for $\alpha_{s}$. There now exist several of these measurements from HERA as well as measurements of $\alpha_{s}$ from inclusive DIS data. At HERA there is also the opportunity to make a measurement of $\alpha_{s}$ at different values of the scale, $Q$ or $E_{T}$ and hence test the running of the value. This is shown in figure 12(a), where the running of $\alpha_{s}$ is seen over a wide range in $E_{T}$ (within one experiment), consistent with the renormalisation group equation. The values of the different methods of measuring $\alpha_{s}$ are shown in figure 12(b) and compared with the world averages. All the values measured are consistent both with each other and the world averages. In particular, the uncertainties on the measurements are also competitive 
with those on the world average. It will be interesting to see the impact these HERA measurements have on future determinations of the world average.
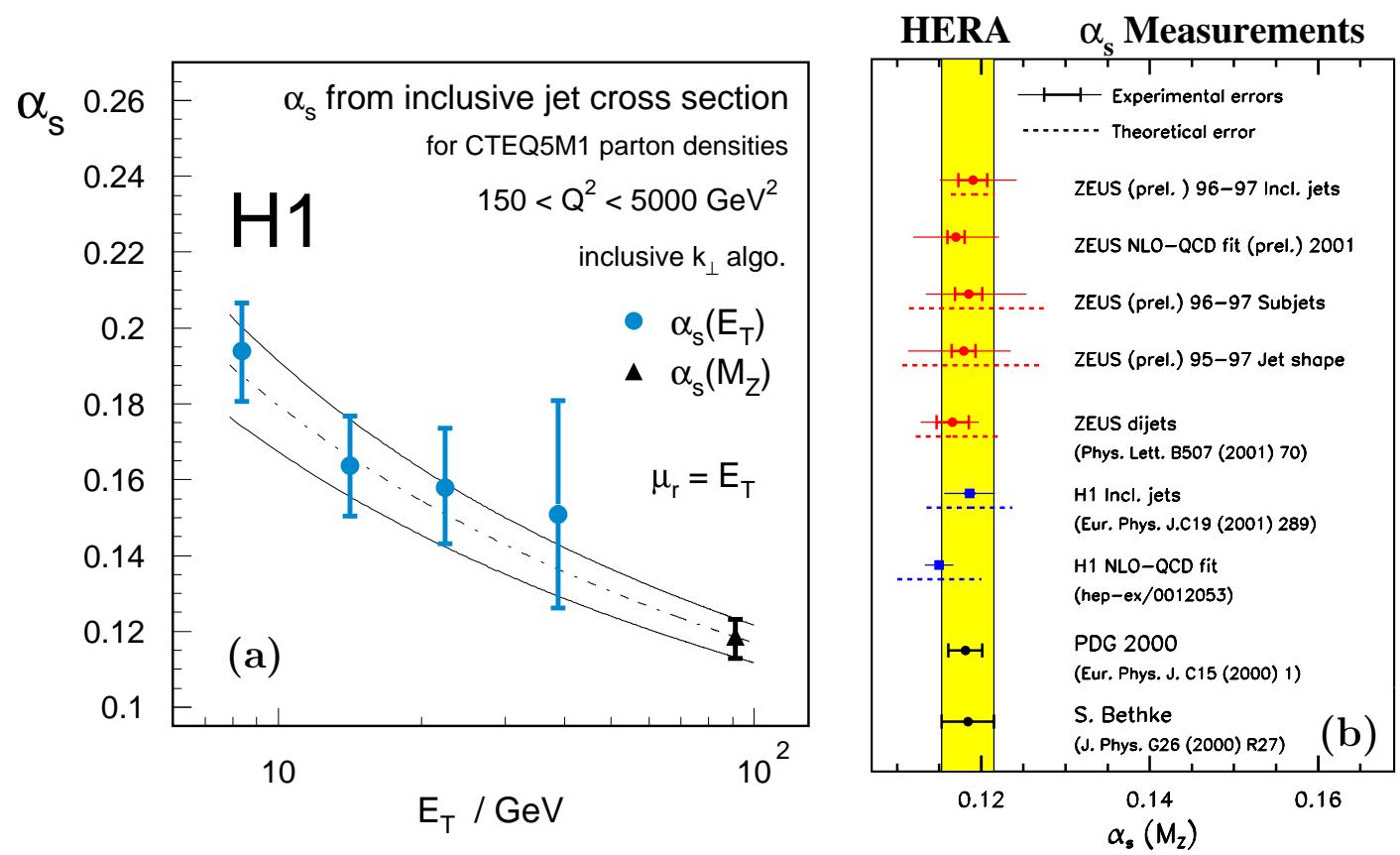

Figure 12. (a) Measurement of $\alpha_{s}$ as a function of the jet transverse energy. (b) Compilation of $\alpha_{s}$-measurements from HERA compared to the world averages.

The measurements of the inclusive jet cross sections at high- $Q^{2}$ show significant potential for the future. The uncertainty associated with that of the renormalisation scale is significantly smaller than in the NLO-QCD fits to $F_{2}^{p}$ data. Using the additional statistics available and those of the future, these measurements could provide very accurate measurements of $\alpha_{s}$. This could also be true for the measurement of the three- to two-jet cross section ratio, with indications of small theoretical uncertainties already observed. The theoretical uncertainties will also be significantly reduced with higher order calculations which are expected soon for fits to $F_{2}^{p}$ data [12] and within a few years for jet calculations 13.

\section{Discussion and summary}

Many measurements of jet production in DIS have been made over a wide kinematic range at HERA. In general, the data are well described by NLO QCD calculations and the current proton parton densities (convoluted with a correction for hadronisation effects), particularly at high scales. From these data, several measurements of $\alpha_{s}$ have been made, all of which agree with the world average and have uncertainties which are also competitive with the world average. 
Although providing good tests of pQCD, further experimental and theoretical work is needed to make really precise comparisons. Most of the data discussed here, use luminosities of the order of $30 \mathrm{pb}^{-1}$. Both experiments currently have about $100 \mathrm{pb}^{-1}$ of data on tape and expect to collect about ten times this amount by the end of HERA II. This significant increase in the data sample will enable high-precision comparisons up to very high- $Q^{2}$ values which are currently statistically limited. Experimentally, the main source of systematic uncertainty arises from uncertainty in the knowledge of the jet energy scale. The current quoted uncertainty is generally $\sim 3-5 \%$, which can produce an uncertainty of $\sim 20 \%$ in the cross section. Efforts are being made (and succeeding) to reduce the uncertainty to $\sim 1-2 \%$, which will lead to an uncertainty in the cross section of $\sim 5 \%$. As most measurements already suffer from dominating theoretical errors, particularly for values of $Q^{2}$ less than $500 \mathrm{GeV}^{2}$, these further experimental improvements will require much more accurate theoretical predictions. At low- $Q^{2}$, the renormalisation scale uncertainty is often greater than $50 \%$, which is too large and hinders conclusions being drawn from the comparison with data, such as the existence of BFKL effects and the need for a resolved photon. Also in this region, the choice of scale is unclear with that of $E_{T}$ seeming to be the natural choice, as $E_{T}>Q$, but with $Q$ appearing to better describe the data. At high- $Q^{2}$, the renormalisation scale uncertainty is still the dominant source of error and its reduction essential for future measurements. There is a strong need for higher order calculations or resummed NLO programs to fully exploit the potential of the measurements being made.

\section{References}

[1] H1 Coll., C. Adloff et al., Euro. Phys. J. C 21 (2001) 1, 33; ZEUS Coll., S. Chekanov et al., accepted by Euro. Phys. J., DESY-01-064.

[2] CDF Coll., T. Affolder et al., Phys. Rev. D 64 (2001) 032001; DO Coll., B. Abbott et al., Phys. Rev. D 64 (2001) 032003.

[3] V. N. Gribov and L. N. Lipatov, Sov. J. Nucl. Phys. 15 (1972) 438 and 675; Yu. L. Dokshitzer, Sov. Phys.-JETP 46 (1977) 641; G. Altarelli and G. Parisi, Nucl. Phys. B 126 (1977) 298.

[4] E. A. Kuraev, L. N. Lipatov and V. S. Fadin, Sov. Phys.-JETP 45 (1977) 199; Ya. Ya. Balitzki and L. N. Lipatov, Sov. J. Nucl. Phys. 28 (1978) 822.

[5] S. Catani and M. H. Seymour, Nucl. Phys. B 485 (1997) 291; erratum ibid B 510 (1997) 503.

[6] H. Georgi and H. D. Politzer, Phys. Rev. Lett. 40 (1978) 3.

[7] ZEUS Collab., J. Breitweg et al., Phys. Lett. B 481 (2000) 199.

[8] B. Pötter, Comp. Phys. Comm. 119 (1999) 45.

[9] M. Botje, Euro. Phys. J. C 14 (2000) 285.

[10] Z. Nagy and Z. Trocsanyi, hep-ph/0104315.

[11] S. Catani et al., Nucl. Phys. B 406 (1993) 187; S. D. Ellis and D. E. Soper Phys. Rev. D 48 (1993) 3160.

[12] W. L. van Neerven and A. Vogt, these proceedings, hep-ph/0107194.

[13] E. W. N. Glover, proceedings of 36th Rencontres de Moriond on QCD and hadronic interactions, Les Arcs, France (2001), hep-ph/0106069. 